\title{
Pain Research Forum: application of scientific social media frameworks in neuroscience
}

\author{
Sudeshna Das ${ }^{1,2 * t}$, Patricia G. McCaffrey ${ }^{3+}$, Megan W. T. Talkington ${ }^{3}$, Neil A. Andrews $^{3}$, \\ Stéphane Corlosquet ${ }^{1}$, Adrian J. Ivinson ${ }^{3}$ and Tim Clark ${ }^{1,2,4}$ \\ ${ }^{1}$ MassGeneral Institute for Neurodegenerative Disease, Massachusetts General Hospital, Cambridge, MA, USA \\ ${ }^{2}$ Department of Neurology, Harvard Medical School, Boston, MA, USA \\ ${ }^{3}$ Harvard NeuroDiscovery Center, Harvard Medical School, Boston, MA, USA \\ ${ }^{4}$ School of Computer Science, University of Manchester, Manchester, UK
}

\section{Edited by:}

Xi Cheng, Lieber Institue for Brain

Development, USA

Reviewed by:

Dimitrios A. Adamos, Aristotle

University of Thessaloniki, Greece

Rochelle Ackerley, University of

Gothenburg, Sweden

Syed Anwar, University of

Engineering and Technology,

Pakistan

\section{*Correspondence:}

Sudeshna Das, MassGeneral Institute for Neurodegenerative

Disease, Massachusetts General Hospital, 65 Landsdowne St.

Cambridge, MA 02139, USA

e-mail: sdas5@mgh.harvard.edu

${ }^{\dagger}$ These authors have contributed equally to this work.
Background: Social media has the potential to accelerate the pace of biomedical research through online collaboration, discussions, and faster sharing of information. Focused web-based scientific social collaboratories such as the Alzheimer Research Forum have been successful in engaging scientists in open discussions of the latest research and identifying gaps in knowledge. However, until recently, tools to rapidly create such communities and provide high-bandwidth information exchange between collaboratories in related fields did not exist.

Methods: We have addressed this need by constructing a reusable framework to build online biomedical communities, based on Drupal, an open-source content management system. The framework incorporates elements of Semantic Web technology combined with social media. Here we present, as an exemplar of a web community built on our framework, the Pain Research Forum (PRF) (http://painresearchforum.org). PRF is a community of chronic pain researchers, established with the goal of fostering collaboration and communication among pain researchers.

Results: Launched in 2011, PRF has over 1300 registered members with permission to submit content. It currently hosts over 150 topical news articles on research; more than 30 active or archived forum discussions and journal club features; a webinar series; an editor-curated weekly updated listing of relevant papers; and several other resources for the pain research community. All content is licensed for reuse under a Creative Commons license; the software is freely available. The framework was reused to develop other sites, notably the Multiple Sclerosis Discovery Forum (http://msdiscovery.org) and StemBook (http://stembook.org).

Discussion: Web-based collaboratories are a crucial integrative tool supporting rapid information transmission and translation in several important research areas. In this article, we discuss the success factors, lessons learned, and ongoing challenges in using PRF as a driving force to develop tools for online collaboration in neuroscience. We also indicate ways these tools can be applied to other areas and uses.

Keywords: social media, neuropathic pain, content management systems, Drupal

\section{INTRODUCTION}

Biomedical scientists rely heavily on the World Wide Web and Internet to do research and to perform literature, database, and information searches. Researchers are also increasingly adopting the Web to collaborate and exchange ideas. Web-based communities that bring together scientists from different disciplines, institutions, and sectors are called "collaboratories" (National Research Council, 1993). Collaboratories with embedded social media tools can increase the pace and quality of scientific collaboration with rapid, open and structured communication (Kouzes et al., 1996; Finholt and Olson, 1997).

However, several challenges for effective collaboration exist with respect to trust, independence, attribution, and intellectual property (Bos et al., 2007; Clark and Kinoshita, 2007). Scientists may prefer to work independently and may be restricted by the boundaries of institutions to freely exchange ideas. Moreover, there is the added complexity of communications across disciplines. Alzforum (http://www.alzforum.org) (Kinoshita and Clark, 2007)—a community of Alzheimer's disease researcherswas successfully able to overcome these challenges with a combination of high quality articles, neutrality, inclusiveness and editorial solicitation/moderation to gain trust and participation (Clark and Kinoshita, 2007). The Schizophrenia Research Forum (SRF) (http://www.schizophreniaforum.org/) was modeled after Alzforum to focus on schizophrenia research. SRF was built using the same software code as the original Alzforum site, and thus has 
an identical look, feel, and functionality to the previous version of Alzforum, before its 2013 re-launch. However, tools to rapidly create and customize such communities were not readily available and therefore the software cost to launch one such community could not be effectively amortized across others using a common software model.

To address this need, we decided to create a reusable platform to build biomedical web communities. We developed the Science Collaboration Framework (SCF) to provide the building blocks for these communities. An earlier version of the platform was developed primarily to publish scholarly articles in biomedicine that can be indexed by the National Library of Medicine (NLM) PubMed library (Das et al., 2009). Our newer version has been re-engineered to add social media and community features similar to those available in Alzforum. We also incorporated elements of Semantic Web technologies (BernersLee et al., 2001) to facilitate interoperability and interdisciplinary communications. Semantic Web is a technology developed by the World Wide Web Consortium (W3C), which aims to promote interoperability of Web content and creation of a "Web of Data" through the use of machine-readable Web pages. It relies heavily upon the used of agreed common vocabularies to describe objects and relationships in the Web. Biomedical science has developed a very rich set of such vocabularies or "ontologies" (over 300 vocabularies with over 5 million terms registered in the National Center for Biomedical Ontology at Stanford University Medical School). Use of ontologies such as these permits, among other benefits, resolution of the many synonym terms in biomedicine, to single common identifiers. Enabling Semantic Web technologies on our framework is meant as a step toward better integration with biomedical vocabularies and databases.

We chose the research area of chronic pain as the first use case. Chronic pain significantly impacts quality of life and is a substantial, growing, and unmet medical need worldwide. Although researchers have made great strides in understanding the underlying mechanisms and neurobiology of pain, few of these discoveries have been translated into new treatments. According to a recent report from the US Institutes of Medicine, chronic pain affects an estimated 100 million people in the US, and costs $\$ 600$ billion annually in health care and lost productivity (National Research Council, 2011); the world-wide toll is unknown. For the most part, chronic pain conditions lack medications that are effective and well tolerated. One of the roadblocks to new treatments is a lack of communication and collaboration between basic, translational, and clinical researchers in the diverse scientific fields and clinical specialties that make up the pain research community. Thus, we developed an online open community, Pain Research Forum (PRF, http://painresearchforum.org), for pain researchers to freely exchange ideas and collectively elevate discussion of the causes of chronic pain and how that knowledge can be translated into new treatments and better care.

\section{MATERIALS AND METHODS}

We have developed a reusable platform-SCF- to build science communities in focused biomedical areas. Previous versions of the platform included tools to publish scientific review articles following the NLM Document Type Definition (DTD), which can be indexed in PubMed (Das et al., 2009). The new release, used for PRF, includes a large number of additional community features, including means to publish news articles, forums, member profiles and various community and research resources. These features are described in the following sections.

\section{ARCHITECTURE}

The SCF is developed on an open-source content management system, Drupal $7^{1}$. Drupal is based on the PHP programming language and MySQL database running in the Linux/Apache web server environment. We chose Drupal because it is easily extensible and there are 30,000 registered Drupal developers continually contributing modules and enhancements to the core features ${ }^{2}$. We developed several custom content types and packaged them as features that can be installed and reused on any science community site. The graphic design (colors, fonts, etc.) is customized for each site using a theme layer (Kumar, 2012). The key content types available in the SCF are described in the next sections. The software is freely available upon request and a complete manual for editors to manage the site is under development.

\section{NEWS ARTICLES AND FORUMS}

The number of papers published in scientific journals continues to grow at a double-exponential rate (Hunter and Cohen, 2006) and it is becoming increasingly difficult for researchers to keep up with the literature. One way to address this problem is to publish news articles that summarize the research and provide context. We have developed a news feature that allows editors of the site to readily publish original news articles on emerging research. The News article has the following main fields: title, subhead, author, and body. The body text is composed in a WYSIWYG editor that allows flexible styling and the ability to add images. News items have references that are implemented as links to bibliographic listings of papers (described in section Papers of the Week). We wanted an easy method for researchers to find relevant news from a certain field, thus news items are categorized with terms from a pre-defined taxonomy. News articles can also be related to other news stories or papers, which appear in a block to the right of the article.

Discussion forums are important social media tools that enable interactions among researchers. Forums have fields similar to those of News. Forums can be Discussions of open research questions, structured Webinars or Journal Clubs. Videos and images can be embedded in Discussion, Webinars, or Journal Clubs. Site editors can specify related items for any Discussion, Webinar, or Journal Club.

Each News article and Forum can be commented on, bookmarked, watched, recommended or shared using social media tools.

\section{PAPERS OF THE WEEK}

Hundreds of papers are published weekly in PubMed for a given biomedical domain such as pain, and editor-curated weekly digests can help researchers stay abreast of the growing literature.

\footnotetext{
${ }^{1}$ http://www.drupal.org

${ }^{2} \mathrm{http}: / /$ en.wikipedia.org/wiki/Drupal
} 
Thus, we decided to create a module for curating and annotating papers downloaded from the NLM PubMed library. We use the previously developed PubMed module to import biomedical articles from PubMed using its Application Programming Interface (API) (Sayers, 2008). The Drupal biblio module ${ }^{3}$ is used to represent papers. We further developed the Journal Stream module that runs nightly queries using the software utility cron and imports the results in batches of 100 items. Complete documentation including a screencast and software for the Journal Stream module is available 4 .

Each imported item is presented to the editors in a moderation queue and can then be "accepted" or "rejected." A screen shot of the moderation queue is shown in Figure 1. The abstract of each paper is displayed so that editors can determine whether the paper should be accepted or rejected. Key papers can be selected as Editors' Picks, and editors can choose to highlight the paper with a few sentences. The accepted papers are published as weekly collections; the periodicity of posting the collections can be configured per site. Once the collection is published, each

\footnotetext{
${ }^{3}$ https://drupal.org/project/biblio

${ }^{4}$ http://scf.github.io/journalstream/
}

paper can be individually commented on, bookmarked, watched, recommended or shared using social media tools. Users may download paper citations into the EndNote reference management software using the Endnote XML format. Currently, only EndNote is available to PRF users, but the Drupal biblio module allows site administrators to enable the BibTeX format if desired, for import into various other reference management tools such as Mendeley, Reference Manager, or Papers.

The citation and Medline Subject Headings (MeSH) terms associated with each paper are automatically updated periodically, as new information is posted in PubMed.

\section{MEMBERS AND REGISTRATION}

Members are the most important component of an online scientific community. We developed tools for members to join the site and publish their profile. While much of the content on the site is freely accessible without registration, only members can post comments on the site and have access to other members' profiles. The registration process starts with the members signing up online using a form. Research credentials including affiliation, position and research interest fields are required. The full name, email, city, and country are also required. Members agree to terms and conditions of membership on the site. The editor is

Papers: 9 Nov 2013 - 15 Nov 2013

\begin{tabular}{|c|c|c|c|c|}
\hline Release Day & Manual & $ت$ & Release Time & $9 a m+1$ \\
\hline Edit Query & & Impor & New Papers & Run Query Now \\
\hline
\end{tabular}

paper review status
Accepted
Not Reviewed
Rejected

Osteoarthritis pain has a significant neuropathic component: an exploratory in vivo patient model. Duarte RV, Raphael JH, Dimitroulas T, Sparkes E, Southall JL, Ashford RL, Kitas GD Rheumatol Int. 2013 Nov 10. PMID: 24212533.

Abstract

Abstract: Osteoarthritis is the most common form of arthritis and includes manifestations of both nociceptive and neuropathic mechanisms. Intravenous lignocaine, a sodium channel blocker and neuronal membrane stabiliser, has been shown in controlled trials to be effective in neuropathic pain; however, the outcome of intravenous lignocaine in osteoarthritis patients has not been assessed yet. The existence of a neuropathic component to the pain of osteoarthritis was investigated by examining possible benefits upon sensory aspects of pain in osteoarthritis patients receiving intravenous lignocaine therapy. Retrospective observational study was carried out using health data routinely collected for non-research purposes. Patients with generalised osteoarthritis who had not responded to more conservative treatments were recruited sequentially and scheduled for intravenous lignocaine therapy either in the rheumatology or pain relief departments. Assessment of efficacy was carried out through a questionnaire including sensory, psychological and social aspects of pain. The sample consisted of 17 women $(60.7 \%$ ) and 11 men $(39.3 \%$ with an average age at the time of treatment of $59 \pm 11$ years. The average pain relief calculated from the NRS scores was $30.2 \pm 21.4 \%$, and the mean duration of pain relief was $10 \pm 6$ weeks. Pain intensity $(p<0.001)$, pain relief $(p<0.003)$ and mobility $(p<0.003)$ were all significantly improved after administration of lignocaine intravenous infusion therapy. Pain was significantly reduced in a group of osteoarthritis patients after administration of intravenous lignocaine. This suggests that part of the pain mechanism in this patient group may be neuropathic, appears to contribute significantly to the patients' pain, and requires further investigation in studies designed specifically for the purpose.

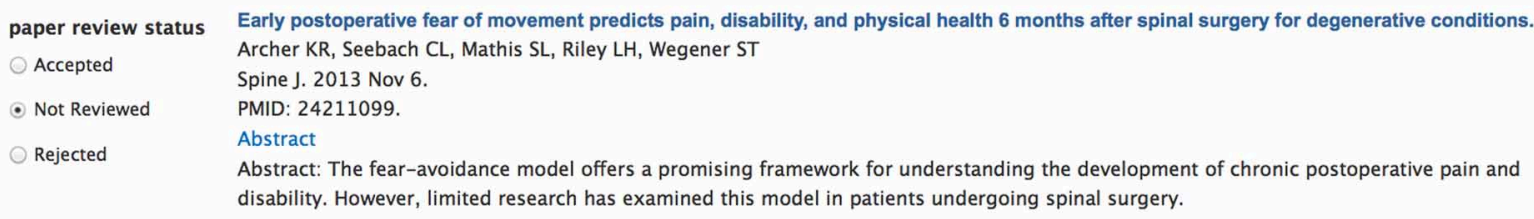

FIGURE 1 | Papers of the Week moderation queue. Papers are imported nightly from NLM PubMed using a tailored query. Editors are presented with an easy-to-use interface to accept or reject the papers. 
notified when a member signs up and once the registration has been reviewed and approved, the new member receives an email informing them of the approval. Email authentication is required for membership activation and reminder emails are sent if members have not responded to the authentication request by 1 week after approval.

Members can publish detailed research profiles on the site, upload their biography, and have the opportunity to import a list of publications directly from PubMed. A member's contributions to the site are also listed on his or her profile page. Members can choose to allow other members to contact them via email functionality provided on the member profile page. Finally, members can subscribe to receive email alerts on new content by type (News, Webinars, Jobs, etc.).

\section{COMMUNITY AND RESEARCH RESOURCES}

We provide a variety of structured resources for members: Meetings and Events, Jobs, Funding Opportunities and Bulletin Board. Meetings and Events allows researchers to quickly find upcoming meetings of interest. These are listed automatically in reverse chronological order. Meetings can be linked to PRF Blogs and News stories on the event, allowing researchers to "catch-up" if they missed the actual event. Jobs provide networking between hiring institutions and applicants; Funding Opportunities highlights grants in the field and Bulletin Board is for posting ad hoc announcements. Together, these community resources provide content tailored to the professional needs of researchers in the pain field. All community resource items have social media tools and can be individually commented on, bookmarked, shared or recommended.

We also provide a variety of tools for creating and publishing research resources. Some are simply pages of information or collections of links to other useful resources. We also developed a structured database for genes associated with a disease or biological condition such as pain. Fields include data to fully describe the gene and details of data on variants associated with pain, with literature references. For studies using research models, the type of model is described, thus presenting a detailed overview of the research done to associate the gene with the disease. In the future, we would also like to create other resources, such as a drugs database that would serve as a central repository for information on new drugs in development and associated clinical trials.

\section{SOCIAL MEDIA TOOLS}

Social media tools are important for online collaboration. We developed or customized a large number of social media tools and incorporated them in our framework. Members can comment on or invite others to comment, share, bookmark, and recommend most content throughout the site. All content on PRF can be emailed and shared on all the popular social network tools (Facebook, Twitter, etc.) by using standard "email" and "share" modules present on every page. RSS news and Twitter feeds are available as well as an email newsletter.

To accommodate the needs of our scientific community, we made a large number of enhancements to the comment feature in Drupal. Scientific commentaries often have attachments or figures, so we developed capabilities for attaching images or documents. The comments can be formatted with a WYSIWYG editor and can be associated with more than one content item if applicable.

\section{WEB SITE USE AND TRACKING}

Websites are tracked using Google Analytics ${ }^{5}$, which provides extensive data on how users interact with the site. We analyze data on number of visits, unique visitors, total pageviews, and views of individual pages. We also look at selected demographic data (country and city of origin), system information, and source of traffic to the site.

\section{SEARCH AND SEMANTIC WEB}

Search is implemented using the open-source enterprise Apache Lucene Solr ${ }^{6}$ search platform. We also implemented sectionspecific searches. Search results can be sorted by date, relevance, number of comments or the date of the last comment. The number of search results per page can be configured by the user. Search results can be filtered using facets. The content type, date, categories etc. are presented as facets.

Semantic Web technologies enable publication of structured documents that can be processed by machines, thus allowing interoperability with the Web of Data (Berners-Lee et al., 2001). We use the Drupal Resource Description Framework Modules (RDF) modules (Corlosquet et al., 2009) to publish RDF of News and Forums. The RDF is indexed and stored in a SPARQL endpoint using the PHP ARC2 libraries ${ }^{7}$. The Dublin Core (Weibel et al., 1998) and Semantically-Interlinked Online Communities (SIOC) (Breslin et al., 2006) ontologies are used to express the RDF. SPARQL queries enable us to perform flexible queries and integrate with other knowledge repositories. Thus, incorporation of Semantic Web technologies in the SCF platform will allow us to network additional online communities built with SCF and identify relevant information across multiple sites.

\section{RESULTS \\ PAIN RESEARCH FORUM}

The SCF platform, originally used for publishing scholarly articles for StemBook (http://stembook.org), was reengineered to create an online community of chronic pain researchers. The goal was to accelerate pain research by enabling free discussion and faster sharing of information between academia, industry, and the clinic, to foster new collaborations and to raise interest in pain research among the wider community of neuroscientists and clinicians. The $\mathrm{PRF}^{8}$ was launched in June 2011; a screenshot of the home page is shown in Figure 2. As of December 2013, PRF has attracted $\sim 1400$ registered members and has published more than 150 News stories and 25 Discussion forums, facilitated five Webinars and published four Journal Clubs features. There are more than 200 member-authored comments on News, Papers, and other content. Papers of the Week are published every Friday and 2-6 papers are highlighted each week as Editors'

\footnotetext{
5 http://www.google.com/analytics/

${ }^{6} \mathrm{http}: / /$ lucene.apache.org/solr/

${ }^{7}$ https://github.com/semsol/arc2

${ }^{8}$ http://www.painresearchforum.org/
} 


\section{PAINRESEARCHFORUM}

Progress through collaboration

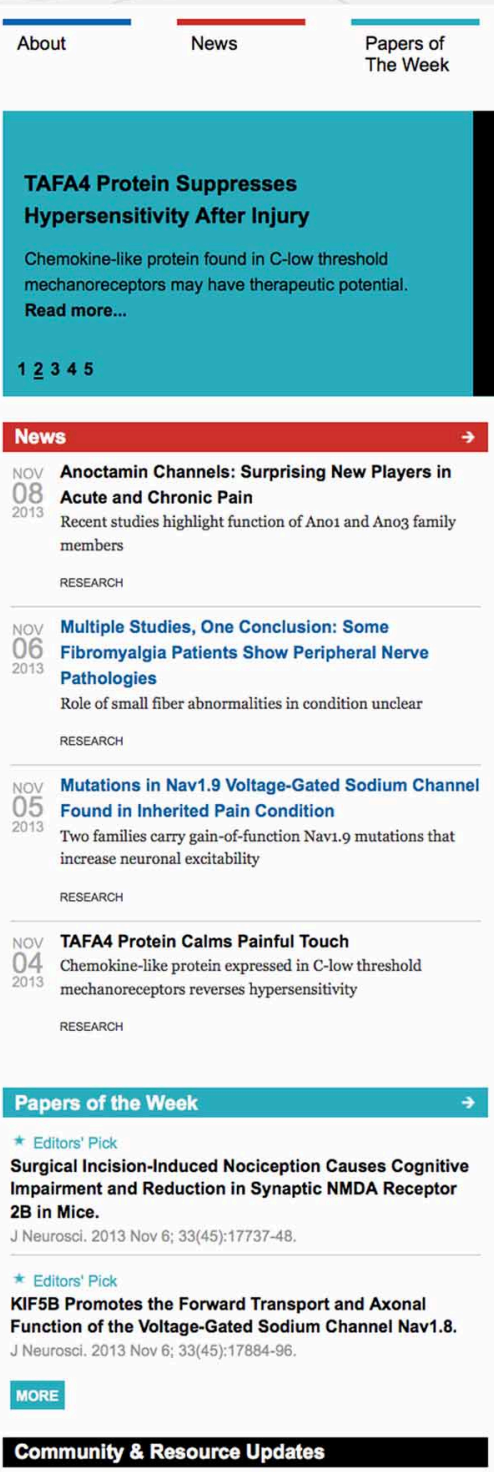

New NIH Funding Opportunity for Small Companies

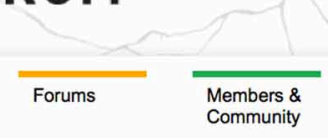

CONTACT | SUPPORT US | NEWSLETTER | RSS FEED \$ | TWITTER
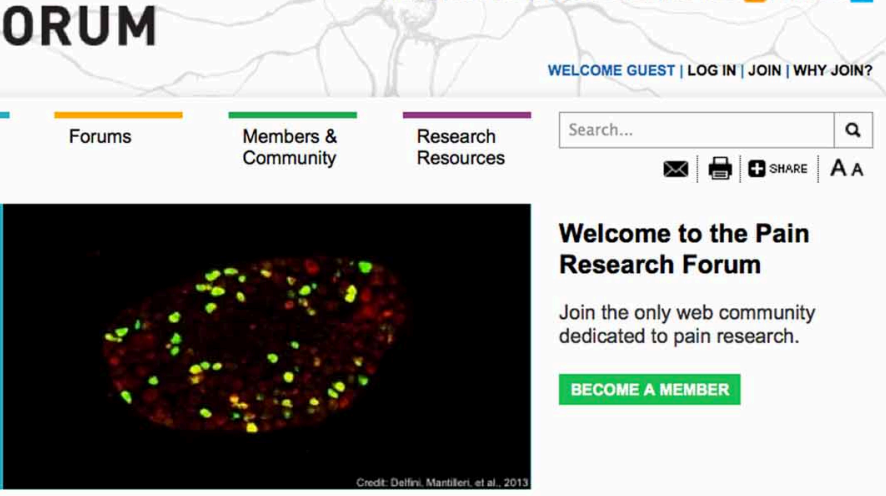

Welcome to the Pain

Research Forum

Join the only web community

dedicated to pain research.

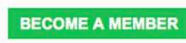

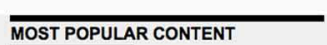

COMMENTED ON VIEWED EMAILED

Brain Activity Shifts as Pain Becomes

Chronic

TMEM16C facilitates $\mathrm{Na}(+)$-activated $\mathrm{K}(+)$

currents in rat sensory neurons and

regulates pain processing.

Unraveling the Mysteries of TRPV1

Regulation

a28-1 Gene Deletion Affects

Somatosensory Neuron Function and Delays Mechanical Hypersensitivity in

Respo Mech to Pipheral Nerve Damage.

Expectation requires treatment to boost

pain relief. An firks stucy.

SEE FULL LIST

\section{Members \& Community $\rightarrow$}

PRF members have full access to other

members' profiles and can contact

colleagues through the site. If you are not a

member yet, it's free and easy to join the

only web community dedicated to pain

research.

Becoming a PRF member demonstrates your support for the Pain Research Forum and will contribute to the site's long-term success.

\section{Recent Comments}

By: Simon van Rysewyk

13 About: Brain Activity Shifts as Pain Becomes Chronic

"Hi Andy, Short answer: yes. I think we should be cautious about what such..."

(1) NEWS

By: John Quintner

13 About: Brain Activity Shifts as Pain Becomes Chronic

"Andrew, the point I am trying to make is that the brain imagery is NOT the..." 8 naws

\section{Research Resources $\rightarrow$}

Information for the new researcher or veteran in the field, pain 101 is our collection of topical articles on important issues in pain research--check out our issues in pain research--check out our
inaugural articles on modeling pain in lower organisms and the role of glia in chronic pain. Our Pain Genes Resource will have the most up-to-date information on the genetics of pain sensitivity, risk of chronic pain and response to analgesics, presented in a format that makes it easy to presented in a format that makes it easy to

FIGURE 2 | Pain Research Forum. Screenshot of home page for Pain Research Forum (http://www.painresearchforum.org) for anonymous users (not logged-in).

Picks. Curated and frequently updated lists of Meetings and Events, Jobs, Funding Opportunities, and Bulletin Board items are posted. The site editors actively curate and maintain several research resources. All content is licensed for reuse under Creative Commons license BY-ND-NC ${ }^{9}$.

\section{NEWS}

PRF publishes 1-2 news stories each week; a screen shot of the News section is shown in Figure 3. News stories are categorized

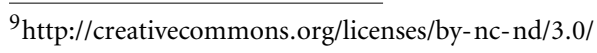

as "Research," "Drug Development," "People" or "Conferences." PRF's news coverage helps researchers stay abreast of the latest findings in the field. For example, PRF was one of the first media outlets to publish a news story about a study of how "high-dose opioid reverses synaptic potentiation in the spinal cord in rats" (Talkington, 2012). The research paper was published in Science on January 13, 2012 and the PRF news story came out 3 days later. Four prominent pain researchers presented their opinions on the work in the form of comments to the story.

Often several related stories are published on an individual topic, and SCF is engineered to allow integration of this material. 


\section{PAINRESEARCHFORUM}

\section{Progress through collaboration}

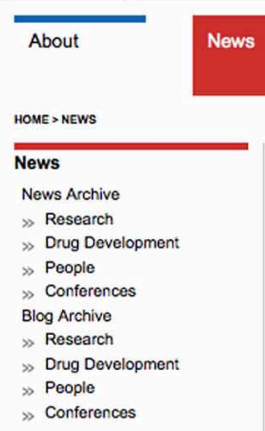

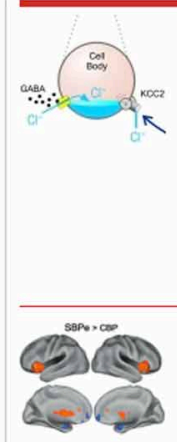

Chloride Transporter Emerges as Druggable Target for Pain

$\mathrm{KCC} 2$-selective compound restores impaired chloride transport in neurons and improves hypersensitivity in rat model of neuropathic pain

Kotz Stephani Sutherland on 16 Oct 2013

The potassium-chloride cotransporter $\mathrm{KCC} 2$ might seem an unlikely target for the next generation of pain medications. The humble protein, found on neurons in the dorsal horn of the spinal...

DRUG DEVEOOMENT | RESENRCH

COMMENT | RECOMMEND

Brain Activity Shifts as Pain Becomes Chronic

Lower back pain signals move from nociceptive regions to areas involved

with emotional aspects of pain

by Abdul-Kareem Ahmed on 8 Oct 2013

Chronic back pain is associated with marked changes in brain activity compared to acute pain, says a new study from A. Vania Apkarian and colleagues at Northwestern University, Chicago,...

RESEARCA

COMMENT | 10 CONMENTS | RECOMMEND

Research Resources: Pain Networks Shows Connections Between
Pain-Related Genes
New online tool provides fresh visual perspective on gene data
by Stephani Sutherland on 7 Oct 2013
With the advent of high-throughput techniques such as microarrays,
researchers have generated mountains of data about genes and proteins
thought to participate in pain processes. But all that information...
RESEARCH
COMMENT | RECOMMEND

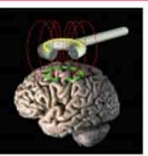

Transcranial Magnetic Stimulation: The Next Wave in Pain Treatment?

Non-invasive technique shows promise but needs more study

by Stephani Sutherland on 3 Oct 2013

Electrical stimulation of the motor cortex was established as an effective treatment for pain 20 years ago, but the risks and drawbacks of surgically implanting electrodes in the brain...

DRUG DEVEOOMNENT | RESERTCH

COUMENT / PECOMUEND

27. $\begin{aligned} & \text { Opioid Receptor Activity Masks Traces of Past Pain Long After Injury } \\ & \text { Interrupting signaling produces withdrawal, hyperalgesia in mice } \\ & \text { by Michele Solis on } 2 \text { Oct } 2013\end{aligned}$
As an injury heals, the dissipation of pain over time is apparently not a
simple return to normal. According to a study published September 20 in
Science, the pain-free...
RESEARCH
COMMENT | RECOMMEND

WELCOME GUEST | LOG IN | JOIN | WHY JOIN?

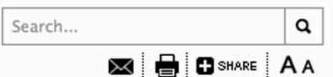

MOST POPULAR NEWS

COMMENTED ON EMAILED VIEWED

Brain Activity Shifts as Pain Becomes

Chronic

Unraveling the Mysteries of TRPV1

Regulation

Multiple Studies, One Conclusion: ( Peripheral Nerve Pathologies

New Optogenetic System Shines a Light on Pain-Sensing Neurons

FLAT-tening Pain

SEE FULL LIST
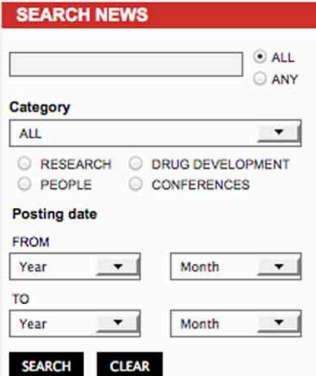

SEARCH CLEAR

FIGURE 3 | News Section in PRF. Screenshot of news section in Pain Research Forum (http://www.painresearchforum.org/news). Five stories are listed per page, social media tools are available for each story. News stories can be filtered using categories on left. Most popular items are highlighted on the right.

For instance, PRF recently covered three high-profile brainimaging studies (Ahmed, 2013; Talkington, 2013; Talkington and McCaffrey, 2013). A screen shot of one of the stories is shown in Figure 4. The forms used to create and publish the story are shown in Figure 5. Related stories are listed in a block on the right. In addition, PRF conducted a webinar in December 2013, featuring one of the principal investigators on the brain imaging papers, along with several panelists including authors of the other papers mentioned in the news coverage. The webinar is also listed as related content to the news story. By hyperlinking, using the References function, cross-posting comments on both news stories and papers, and using the Related Content feature, PRF is able to provide a contextualized, intelligent overview of fast-moving developments in this corner of the larger field. Social media tools 


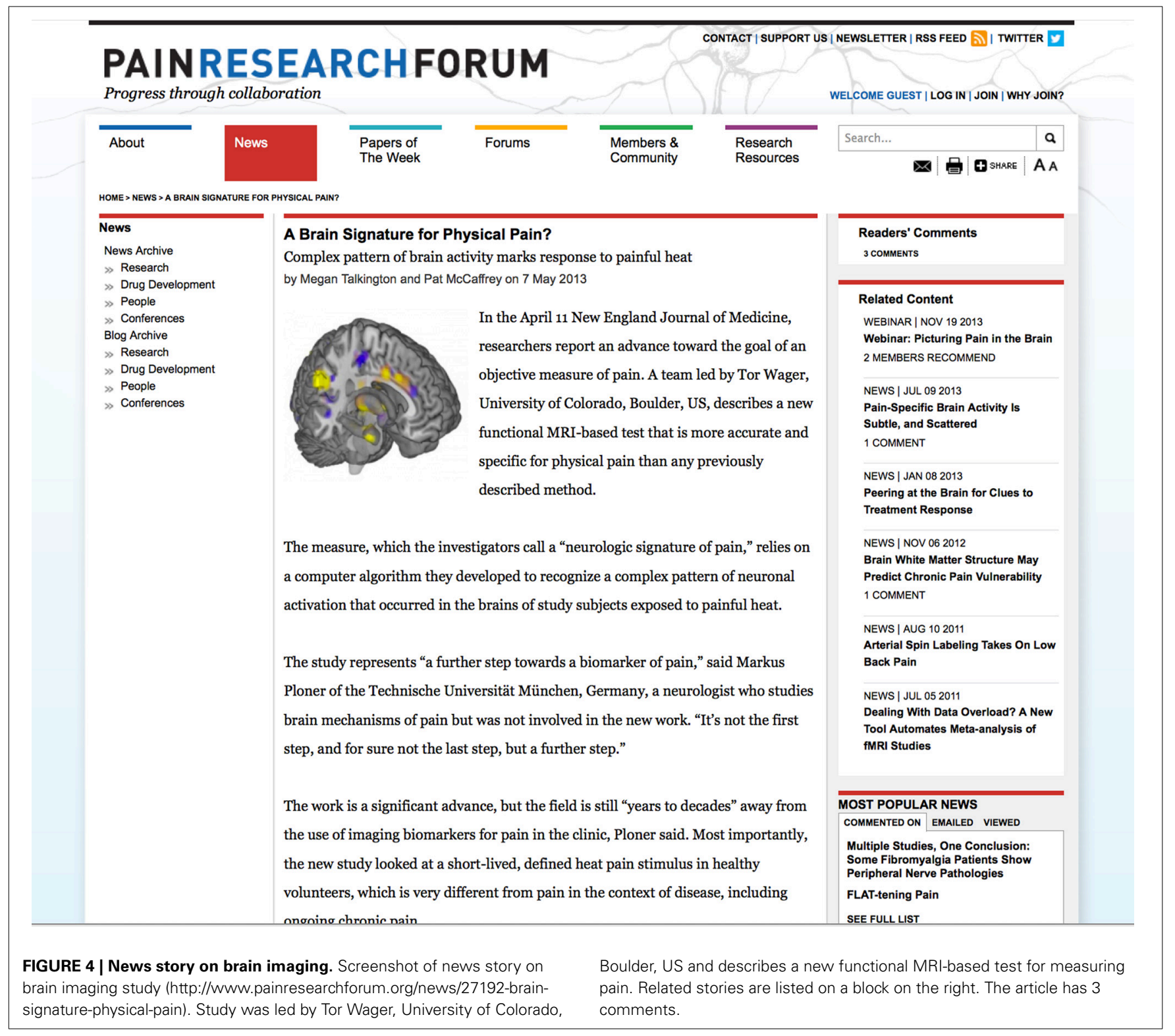

such as Twitter feeds and newsletters disseminate the information quickly and effectively to members.

Brain imaging in the context of understanding and detecting pain is a popular but controversial topic, and the three stories cited above (Ahmed, 2013; Talkington, 2013; Talkington and McCaffrey, 2013) elicited several comments from PRF members. It is significant that many of the researchers commenting on PRF are junior people, including graduate students or postdoctoral fellows. Often the study authors participate in the discussion: for example, on the news story by Talkington and McCaffrey (2013), the study authors responded to two previous comments from researchers not involved with the study. Thus, the commenting feature on PRF news stories serves a function similar to the "letters to the editor" sections of journals, with key differences: it is faster, has a lower barrier to entry, and welcomes contributions from junior researchers.
Currently, the most accessed news story is one that discusses the new research on the use of antibiotics to relieve some forms of chronic lower back pain (Morton, 2013). The PRF news story covers two published research studies: one suggests that pain may be caused by a low-grade bacterial infection in the discs and the other finds that antibiotics can effectively treat the pain and prevent further tissue degeneration. Both studies have implications for patients with long-standing low back pain, and they elicited a lot of attention and controversy including in the popular press. In a Google search for the query "antibiotics for back pain," the PRF news story is the number one hit. This shows that PRF news stories can be highly ranked in Google searches for general pain terms, giving many readers access to the site. This high ranking may contribute to the unusually high number of page views for this article, which are three times more than the second most viewed news article. 


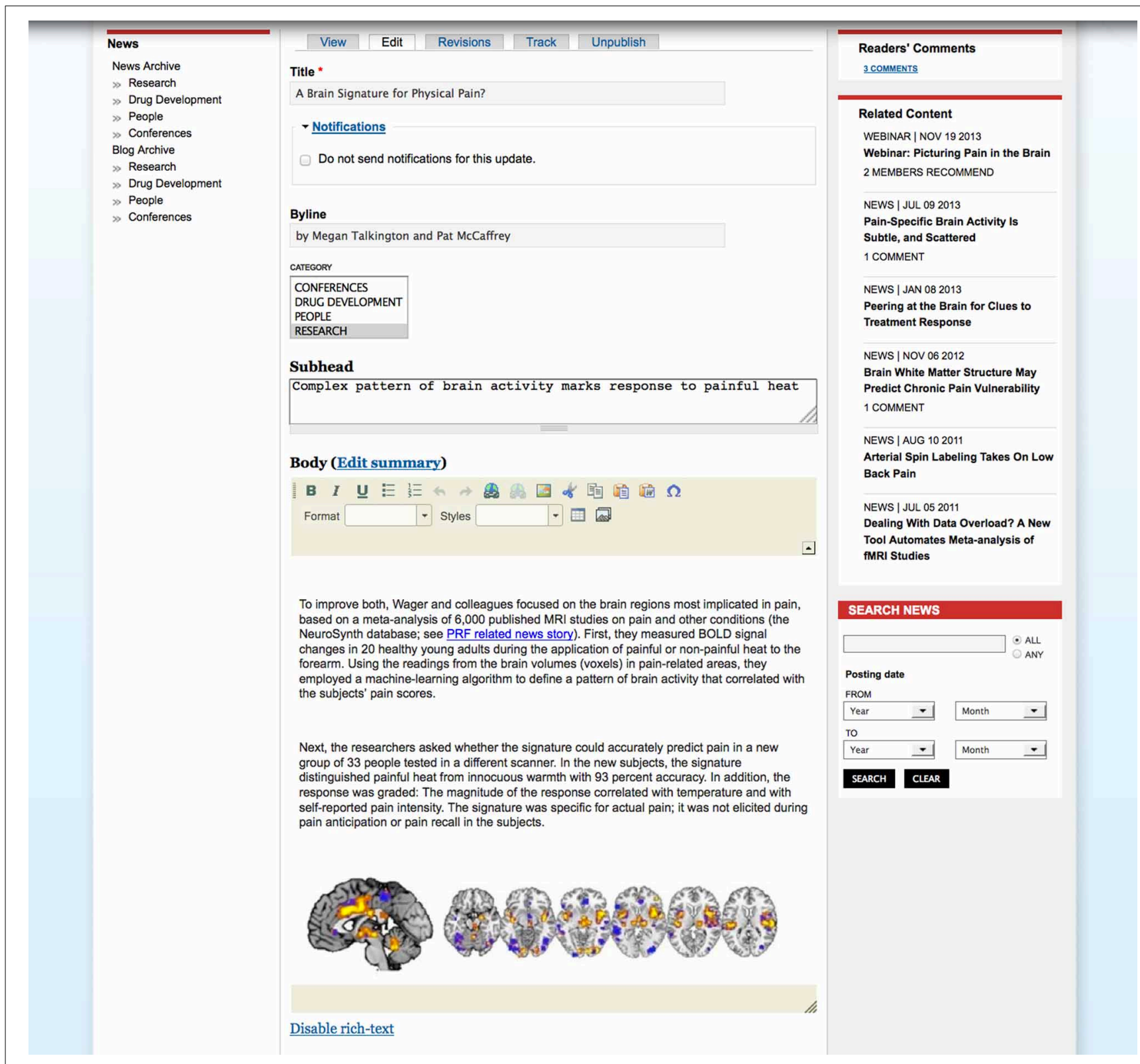

FIGURE 5 | Forms used by editors for a News story. Screenshot of forms used by the editors to create and publish a news story. A form is available for each field and the body is composed using a WYSIWYG editor.

\section{FORUMS}

The PRF Forums category includes Discussions, Webinars, and Journal Clubs. PRF editors have moderated several online discussions. The most-accessed Discussion is a debate on how the human brain processes stimuli, initiated by a well known pain researcher and PRF Science Advisory Board member (Basbaum, 2011). A Discussion on the challenges associated with translating pain research discoveries into clinical developments, presented by another researcher and science advisor, attracted many follow-on comments (Mogil, 2011). In 2013, PRF conducted five Webinars, each typically attracting $\sim 150$ registered attendees, plus an unknown number of additional viewers who watched the event in groups under one registration. Each Webinar is conducted online using a webinar-hosting service and a recording is subsequently posted to PRF with a written introduction. This archives the presentation for future viewing and enables an ongoing, online conversation beyond the duration of the actual presentation. The Journal Club is a venue for disseminating the results of discussions that occur in individual lab groups about recently published scientific articles. For example, a graduate student and postdoctoral fellow studying pediatric pain presented a journal club at their institution on Walker et al. (2012; Birnie and Caes, 2012). They then wrote for PRF a brief synopsis of the study and the discussion that took place in their meeting. They also posed questions to the author of the original paper, who responded with her own 
online comment. A screenshot of the Journal Club is shown in Figure 6.

\section{PAPERS OF THE WEEK}

Papers of the Week are published every Friday and provide a curated digest of recent and noteworthy pain-relevant articles published in academic journals. For example, for the November $2-8,2013$ collection $^{10}, 61$ papers were identified and listed. Two were further highlighted as Editors' Picks. Highlighted papers are often commented on by PRF-registered members, sometimes resulting in vibrant back-and-forth discussions between the authors and other PRF members ${ }^{11}$. Related papers are listed in a block on the right as shown in Figure 7; a news story on the paper

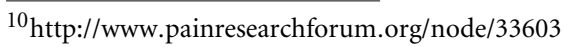

${ }^{11} \mathrm{http}: / /$ www.painresearchforum.org/papers/22720-activation-5-ht2areceptors-upregulates-function-neuronal-k-cl-cotransporter-kcc2
}

is listed under the paper citation. Papers of the Week are archived as weekly lists, and the database of all papers can be searched with a detailed section-specific search. Members can also search for other papers using links provided on the paper page to Google Scholar or PubMed.

\section{PRF MEMBERSHIP AND USAGE STATISTICS}

As of December 17, 2013, PRF has just over 1400 registered members, of whom 1143 have published profiles in the member directory. Member demographics are shown in Figure 8. A variety of professionals sectors are represented including universities, hospitals, industry, government, and non-profit foundations, with the majority of members coming from academia (Figure 8A). Most members are research scientists and academicians including graduate students and postdoctoral fellows (Figure 8B). Twothirds $(67 \%)$ of PRF members have an advanced degree, $41 \%$ have earned $\mathrm{ahD}$ and $20 \%$ have an MD or DDS.

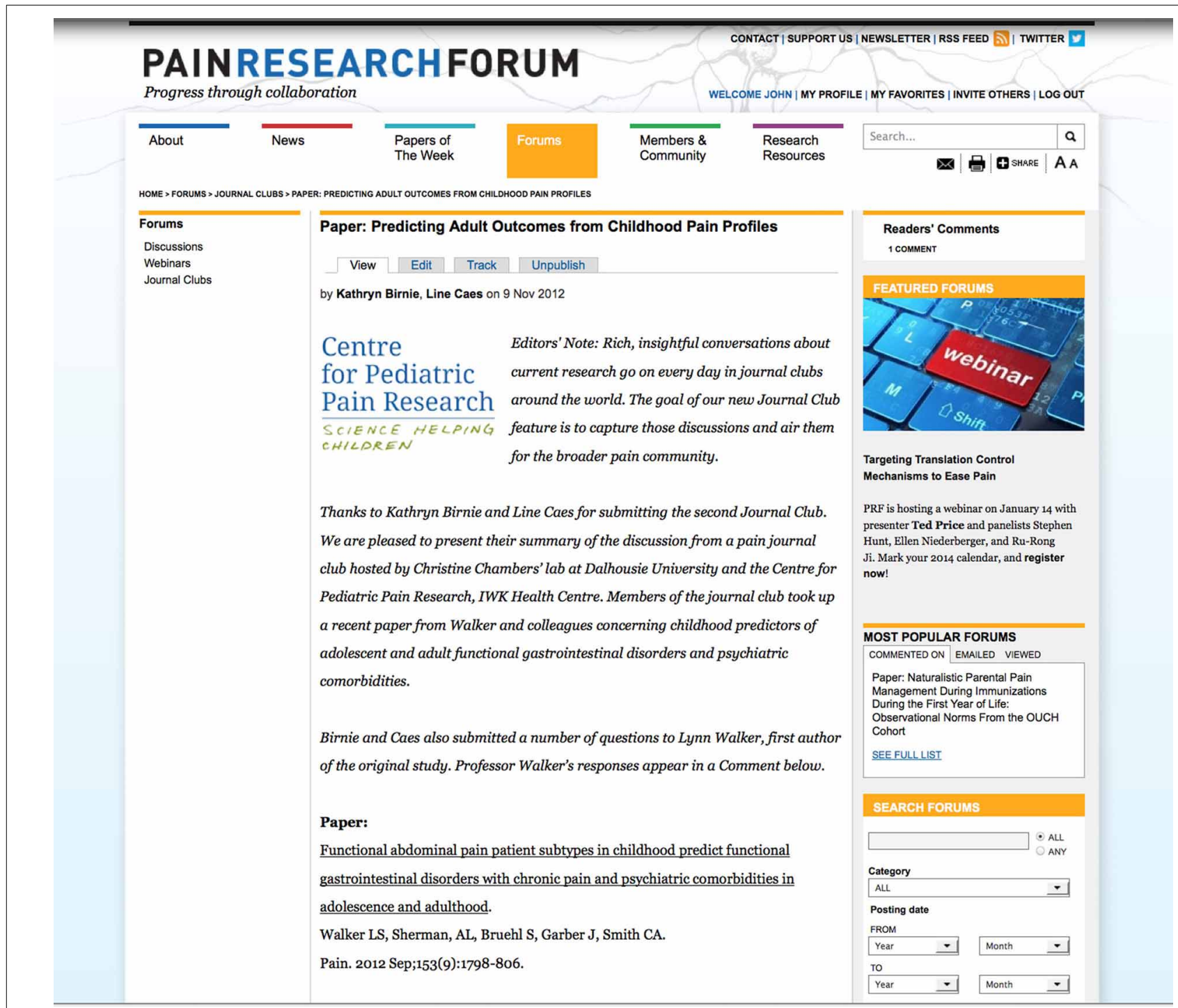

FIGURE 6 | Journal Club on recently published paper. Screenshot of journal club featuring a recently published paper in the journal PAIN (http://www. painresearchforum.org/forums/journal-club/21586-predicting-adult-outcomes-childhood-pain-profiles). Featured forums are highlighted in a block on the right. 


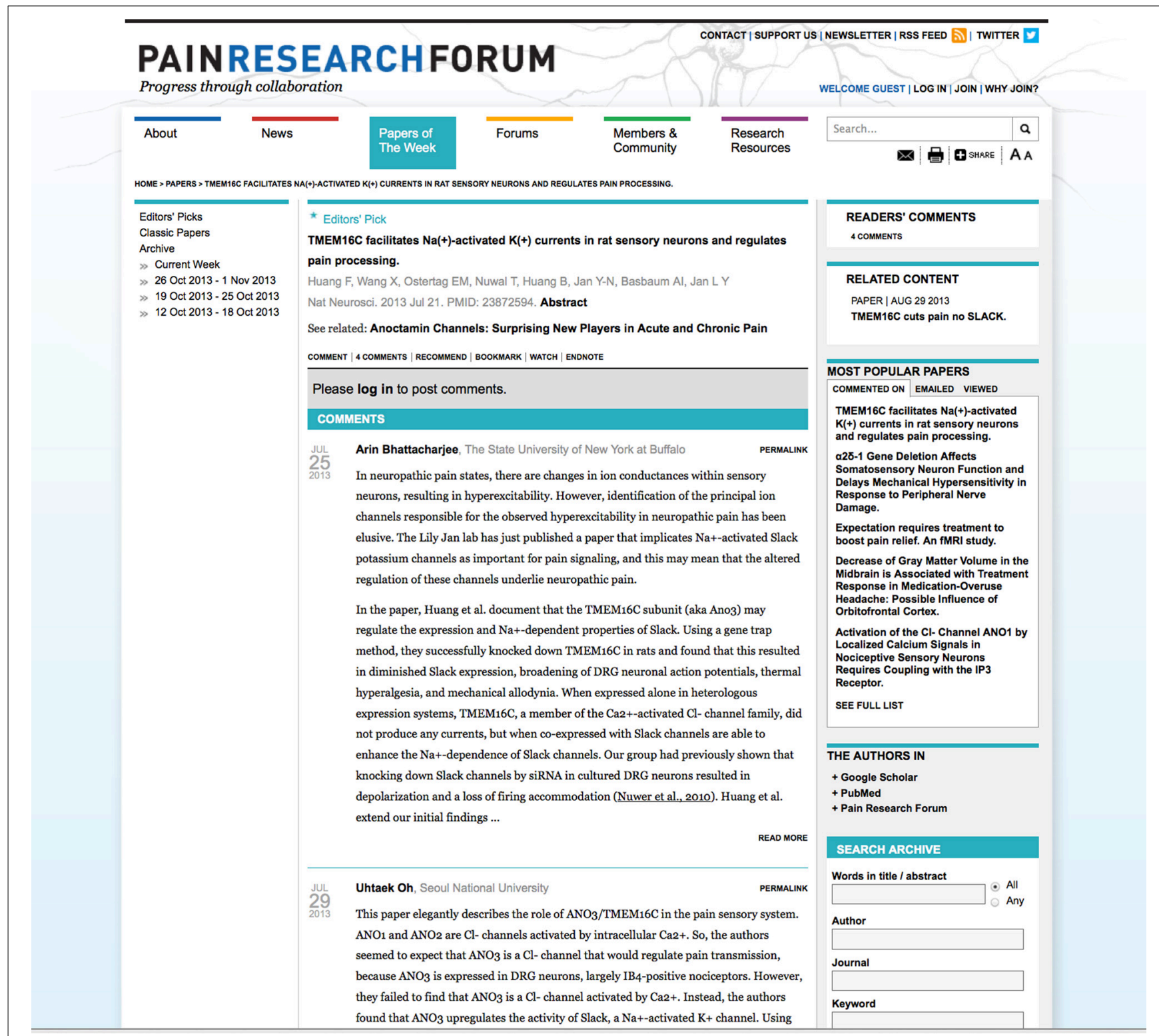

FIGURE 7 | Sample Paper from Papers of the Week. Screenshot of paper published in Papers of the Week (http://www.painresearchforum.org/papers/29791tmem16c-facilitates-na-activated-k-currents-rat-sensory-neurons-and-regulates-pain). Related paper is listed on the right. Section specific search is also available.

Although promotional efforts—emails, conference attendance, printed literature, etc.-have been responsible for attracting many members (Figure 8C), more than half of members found PRF either via a Web search, word of mouth or were directly invited to join by an existing member. Social media tools played an important role in recruiting members ("Other" Category).

We found that a significant proportion (about one-third) of newly registered members failed to respond to the email validation message, which asked them to click a link and return to the site to complete the registration process. We installed a module to automatically send reminder emails to these new members, and have recovered about half of the non-responders.
Google Analytics shows that in October 2013, PRF had $\sim 8000$ unique visitors, $\sim 11,000$ visits and over 25,000 page views. PRF has visitors from all over the world, with the majority from the USA, UK, Canada, and Australia. The most popular browsers are Chrome, Safari, and Firefox. The Papers of the Week and News are the most popular sections; popular community pages include Jobs and Meetings \& Events. Some of the news stories mentioned above are among the top pages visited on the site.

\section{COMMUNITY AND RESEARCH RESOURCES}

PRF lists community and research resources of interest to the community, and the software automatically highlights new listings on the home page and section landing pages. Currently users 


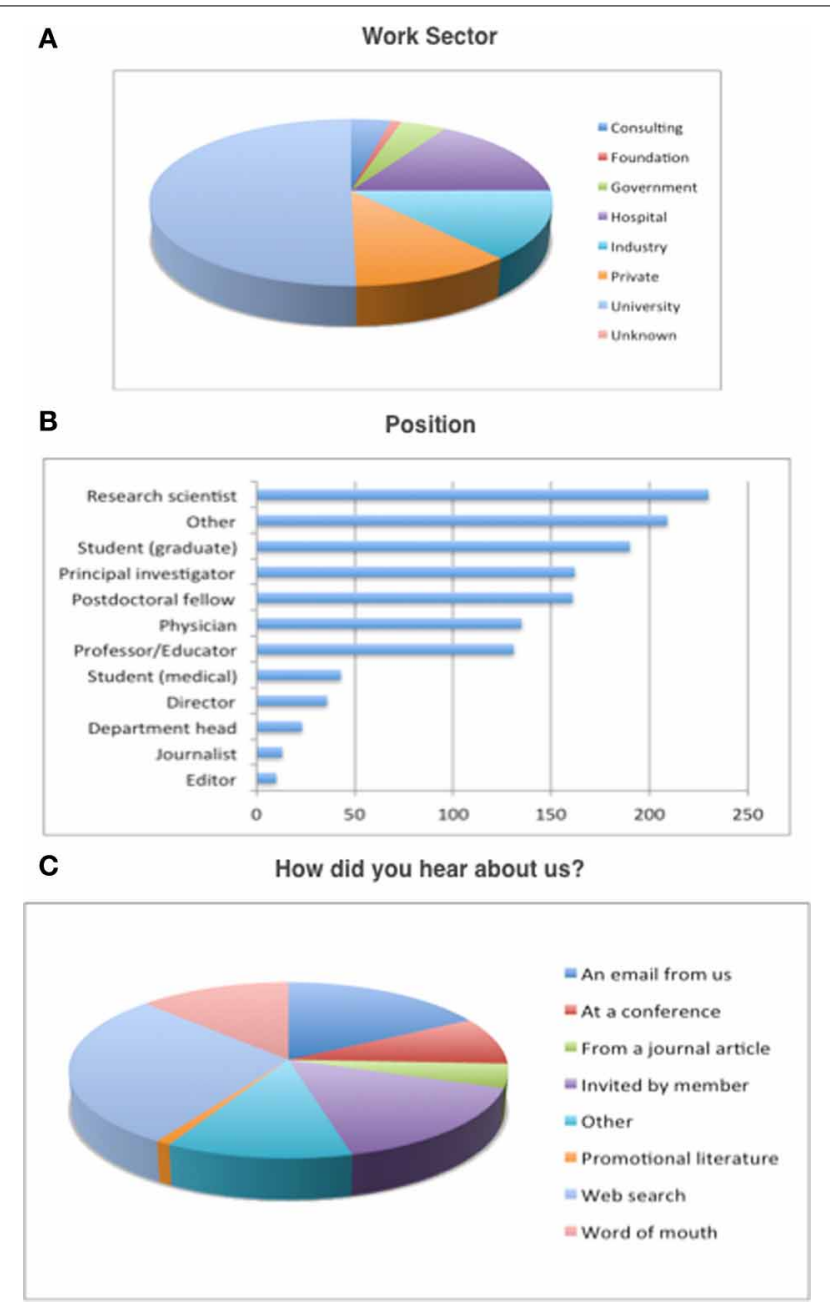

FIGURE 8 | Membership Statistics. (A) Shows the distribution of work sectors, (B) shows the different position of members, and (C) depicts the how different members heard about PRF.

can access information on more than 30 upcoming meetings and read coverage of past meetings. Job postings ${ }^{12}$, funding opportunities $^{13}$, and bulletin board ${ }^{14}$ items are posted. In addition, several research resources are also provided including a curated pain gene resource consisting of about 25 genes that are associated with pain, according to peer reviewed, published studies, and a collection of "Pain 101" articles covering basic questions in pain research. A collection of useful links is planned.

\section{SEARCH AND SEMANTIC WEB}

We have implemented a general as well as section-specific search for PRF using the Apace Solr module as described in the Methods section. The search results can be further filtered by content type, date, news topic or whether an item is recommended or has comments (Figure 9). The search term is highlighted in the results and

\footnotetext{
${ }^{12} \mathrm{http} / / /$ www.painresearchforum.org/members/jobs

${ }^{13} \mathrm{http} / / / \mathrm{www}$.painresearchforum.org/members/funding-opportunities

${ }^{14} \mathrm{http} / / /$ www.painresearchforum.org/members/bulletin-board
}

users have the option to sort the results by type, date, number of comments or last comment date.

We have created Linked data and RDF for News, Forums, and Papers using the Drupal RDF modules along with the SIOC and DC ontologies. A sample RDF ${ }^{15}$ illustrates the use of these ontologies to describe the News article. The RDF is indexed using the ARC2 PHP libraries and is available at http://www. painresearchforum.org/sparql. The SPARQL endpoint allows us to perform flexible queries such as "all News articles published with greater than 2 comments" (see Table 1). In the future, we could perform federated queries across endpoints from multiple communities.

\section{DISCUSSION}

Our reusable platform, SCF, was successfully deployed to create a vibrant online community of chronic pain researchers: PRF. In a little over 2 years, PRF has attracted a large community of registered users and contributors including members from academia, industry, government, and non-profit organizations. Scientists engaged in laboratory research, clinicians, students, and fellows are all represented. PRF is a network of investigators from various sectors and disciplines and a venue for discussing, critiquing, and advancing pain research. The response to PRF in terms of member registrations and site use indicates a pent-up demand for such online communities that provide researchers in diseasecircumscribed fields of biomedical research with news, forums, and resources that are most relevant to them in one place.

In our experience, editorial involvement is crucial to keep the site active and vibrant with user interactions. Reporting news, moderating discussions, soliciting comments and producing webinars and journal club pieces is labor intensive but necessary to maintain high quality interactions within the user community. On PRF, this is accomplished by a staff of professional editors and writers with backgrounds in research and neurobiology, whose primary responsibility is to create and moderate content. Assembling an active and engaged Scientific Advisory Board made up of leading researchers and clinicians from a variety of disciplines is also important to ensure the highest quality content, provide community outreach and promote community involvement.

PRF is publicized in the pain research community by several avenues. The launch was announced with a press release and with a direct email to several thousand pain researchers, identified through meeting rosters, publications, and a existing pain research listserve. In addition, fliers were distributed at pain meetings and neuroscience meetings, including 7000 postcards placed in meeting bags at the most recent World Congress on Pain in Milan (2012), the largest gathering of pain researchers in the world. Several pain professional groups promote the site to their members on their websites or in member newsletters. PRF editors have given talks and posters at pain conferences. The PRF science advisors promote the site to their colleagues using slides and other materials provided by PRF. The site is also marketed through word of mouth, a monthly email newsletter, and RSS and Twitter (@PainResForum) feeds.

\footnotetext{
${ }^{15} \mathrm{http} / / /$ www.painresearchforum.org/node/34289.rdf
} 


\section{PAINRESEARCHFORUM}

Progress through collaboration

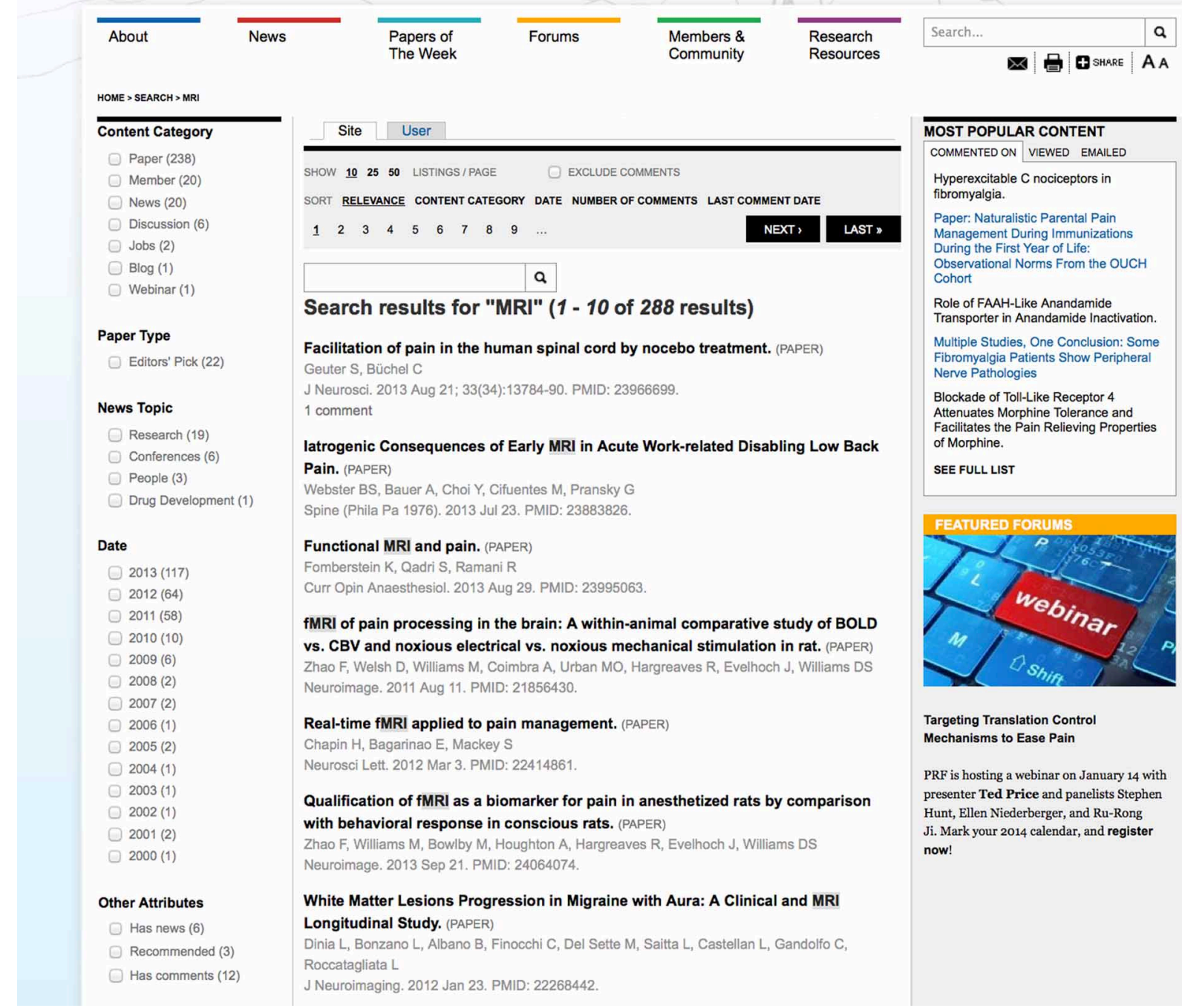

CONTACT | SUPPORT US | NEWSLETTER | RSS FEED B | TWITTER V

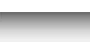

FIGURE 9 | Search Results. This figure displays the search results for the term MRI. The search results can be filtered using various facets on the left and sorted by content type, date, number of comments or date of last comment.

\section{Table 1 | Example SPARQL Query.}

PREFIX schema: <http://schema.org/>

PREFIX sioc: <http://rdfs.org/sioc/ns\#>

SELECT ?post ?title ?replies

WHERE \{

?post a schema:NewsArticle;

schema:name ?title;

sioc:num_replies ?replies.

FILTER (\$replies > 2)

\}

ORDER BY DESC(?replies)

Query to find news articles with $>2$ comments.
One barrier to progress in research is the reticence of researchers to divulge unpublished or other preliminary work, or to publicly criticize the work of others. Web communities like PRF provide a new model of open communication that will help change this culture and promote faster and freer information exchange. A barrier to achieving more researcher involvement in and contributions to communities like PRF is the lack of incentives for scientists to contribute comments or other materials that do not add to their official publication record. In the future, sites like PRF should aim to provide incentives for contribution, for example by indexing content on PubMed or by arranging to provide continuing medical education (CME) credits for physicians who contribute. 
All content on PRF is provided free of charge to the research community, and funding of shared resources like PRF is an ongoing challenge. To attract donors and other sponsors of the site we must continually demonstrate both scientific credibility in all of the content presented, and constant, lively and intellectually valuable interactions. Consistent outreach to and education of potential funders in the philanthropic area, professional societies, relevant pharmaceutical and biotech companies and the academic sector is also required. At the same time, editorial independence from sponsors must be strictly maintained. PRF does not accept paid advertisements and does not intend to do so.

In terms of technology, the SCF platform consists of a comprehensive set of building blocks for an online community. We have effectively used the SCF platform to create other communities: the Multiple Sclerosis Discovery Forum (msdiscovery.org) and StemBook (stembook.org). The platform incorporates elements of Semantic Web technologies, which have the potential to accelerate the pace of inter-disciplinary research by defining a common language and improving interoperability between various resources on the Web (Hendler, 2003). In the future, we plan to leverage these Semantic Web technologies to enable us to do cross-site queries and find relevant information on other sites. Interoperability between these multiple communities involved in neurobiology and neurology research will, we hope, identify common biological mechanisms behind complex neurological diseases and accelerate translation of science to new treatments.

\section{ACKNOWLEDGMENTS}

We gratefully acknowledge essential funding from the Curvey Family Foundation, the Mayday Fund, the Guthart Family Foundation, Purdue Pharma L.P. and Genentech. We thank Alzforum and PDOnline editors for their feedback and ideas, Nick Maloney, Paul Northup, Jake Strawn, and Chris Akeley for their contributions to software development, and Stoltze Design for graphic design.

\section{REFERENCES}

Ahmed, A. K. (2013). Brain Activity Shifts as Pain Becomes Chronic [Online]. Pain Research Forum. Available online at: http://www.painresearchforum.org/news/ 32409-brain-activity-shifts-pain-becomes-chronic (Accessed 2013).

Basbaum, A. (2011). Specificity Versus Patterning Theory: Continuing the Debate [Online]. Pain Research Forum. Available online at: http://www.painresearchforum.org/forums/discussion/7347-specificity-versuspatterning-theory-continuing-debate (Accessed 2013).

Berners-Lee, T., Hendler, J., and Lassila, O. (2001). The semantic web. Sci. Am. 284, 28-37. doi: 10.1038/scientificamerican0501-34

Birnie, K., and Caes, L. (2012). Paper: Predicting Adult Outcomes from Childhood Pain Profiles [Online]. Pain Research Forum. Available online at: http://www.painresearchforum.org/forums/journal-club/21586-predictingadult-outcomes-childhood-pain-profiles (Accessed 2013).

Bos, N., Zimmerman, A., Olson, J., Yew, J., Yerkie, J., Dahl, E., et al. (2007). From shared databases to communities of practice: a taxonomy of collaboratories. J. Comput. Mediat. Comm. 12, 652-672. doi: 10.1111/j.1083-6101.2007.00343.x

Breslin, J. G., Decker, S., Harth, A., and Bojars, U. (2006). SIOC: an approach to connect web-based communities. Int. J. Web Based Commun. 2, 133-142. doi: 10.1504/IJWBC.2006.010305

Clark, T., and Kinoshita, J. (2007). Alzforum and SWAN: the present and future of scientific web communities. Brief. Bioinform. 8, 163-171. doi: 10.1093/bib/bbm012

Corlosquet, S., Delbru, R., Clark, T. W., Polleres, A., and Decker, S. (2009). "Produce and consume linked data with drupal," in 8th International Semantic Web Conference (ISWCC) (Washington, DC).
Das, S., Girard, L., Green, T., Weitzman, L., Lewis-Bowen, A., and Clark, T. (2009). Building biomedical web communities using a semantically aware content management system. Brief. Bioinform. 10, 129-138. doi: 10.1093/bib/ bbn052

Finholt, T. A., and Olson, G. M. (1997). From laboratories to collaboratories: a new organizational form for scientific collaboration. Psychol. Sci. 8, 28-36. doi: 10.1111/j.1467-9280.1997.tb00540.x

Hendler, J. (2003). Communication. Science and the semantic web. Science 299, 520-521. doi: 10.1126/science.1078874

Hunter, L., and Cohen, K. B. (2006). Biomedical language processing: what's beyond PubMed? Mol. Cell 21, 589-594. doi: 10.1016/j.molcel.2006.02.012

Kinoshita, J., and Clark, T. (2007). Alzforum. Methods Mol. Biol. 401, 365-381. doi: 10.1007/978-1-59745-520-6_19

Kouzes, R. T., Myers, J. D., and Wulf, W. A. (1996). Collaboratories: doing science on the internet. Computer 29, 40-46. doi: 10.1109/2.532044

Kumar, K. (2012). Drupal 7 Theming Cookbook. Birmingham: Packt Publishing Ltd.

Mogil, J. (2011). What Is the Reason for Lack of Translation in the Pain Field? [Online]. Pain Research Forum. Available online at: http://www. painresearchforum.org/forums/discussion/4561-what-reason-lack-translation-pain-field (Accessed 2013).

Morton, C. C. (2013). Antibiotics May Relieve Some Chronic Low Back Pain, Study Suggests [Online]. Pain Research Forum. Available online at: http://www.painresearchforum.org/news/28081-antibiotics-may-relieve-somechronic-low-back-pain-study-suggests (Accessed 2013).

National Research Council, USA. (1993). National Collaboratories: Applying Information Technology for Scientific Research. Washington, DC: The National Academy Press.

National Research Council, USA. (2011). Relieving Pain in America: A Blueprint for Transforming Prevention, Care, Education, and Research. Washington, DC: The National Academies Press.

Sayers, E. (2008). E-utilities Quick Start. Bethesda, MD: National Center for Biotechnology Information.

Talkington, M. (2012). Erasing the Spinal Memory of Pain? [Online]. Pain Research Forum. Available online at: http://www. painresearchforum.org/news/12689-erasing-spinal-memory-pain (Accessed 2013).

Talkington, M. (2013). Pain-Specific Brain Activity is Subtle, and Scattered [Online]. Pain Research Forum. Available online at: http://www.painresearchforum. org/news/29430-pain-specific-brain-activity-subtle-and-scattered (Accessed 2013).

Talkington, M., and McCaffrey, P. G. (2013). A Brain Signature for Physical Pain? [Online]. Pain Research Forum. Available online at: http://www. painresearchforum.org/news/27192-brain-signature-physical-pain (Accessed 2013).

Walker, L. S., Sherman, A. L., Bruehl, S., Garber, J., and Smith, C. A. (2012). Functional abdominal pain patient subtypes in childhood predict functional gastrointestinal disorders with chronic pain and psychiatric comorbidities in adolescence and adulthood. Pain 153, 1798-1806. doi: 10.1016/j.pain.2012. 03.026

Weibel, S., Kunze, J., Lagoze, C., and Wolf, M. (1998). Dublin core metadata for resource discovery. Internet Eng. Task Force RFC 2413, 222.

Conflict of Interest Statement: The authors declare that the research was conducted in the absence of any commercial or financial relationships that could be construed as a potential conflict of interest.

Received: 24 December 2013; accepted: 19 February 2014; published online: 11 March 2014.

Citation: Das S, McCaffrey PG, Talkington MWT, Andrews NA, Corlosquet S, Ivinson AJ and Clark T (2014) Pain Research Forum: application of scientific social media frameworks in neuroscience. Front. Neuroinform. 8:21. doi: 10.3389/fninf.2014.00021 This article was submitted to the journal Frontiers in Neuroinformatics.

Copyright (c) 2014 Das, McCaffrey, Talkington, Andrews, Corlosquet, Ivinson and Clark. This is an open-access article distributed under the terms of the Creative Commons Attribution License (CC BY). The use, distribution or reproduction in other forums is permitted, provided the original author(s) or licensor are credited and that the original publication in this journal is cited, in accordance with accepted academic practice. No use, distribution or reproduction is permitted which does not comply with these terms. 